\title{
Evaluación neuropsicológica en niños y adolescentes con fenilcetonuria e hiperfenilalaninemia y su relación con los niveles de fenilalanina plasmática Neuropsychological assessment among children and adolescents with phenylketonuria and hyperphenylalaninemia and its relationship with plasma phenylalanine levels
}

Dra. María B. González García, Dr. Pablo Conde-Guzon ${ }^{b}$ Lic. Carlos Alcalde Martín Dra. María J. Conde-Guzon ${ }^{b}$ y Dr. Roberto Velasco Zúñiga

\section{RESUMEN}

Aunque, con tratamiento precoz, los pacientes con fenilcetonuria pueden presentar niveles de inteligencia normales, es importante optimizar el control dietético para mantener niveles de fenilalanina adecuados y poder desarrollar su potencial intelectual sin alteraciones en sus tareas diarias por déficits en las funciones ejecutivas. Se presenta una serie de 26 pacientes, diagnosticadosy tratados precozmente, a quienes se realizó una evaluación psicométrica junto con determinaciones de fenilalanina a lo largo de su vida y en el momento de realización de los tests. Se observa una tendencia a la relación inversa entre el cociente intelectual y la fenilalanina concurrente, la mediana de fenilalanina y el cociente fenilalanina/tirosina, así como una tendencia a la relación negativa entre las funciones ejecutivas y los valores de fenilalanina concurrentes y durante la vida.

Palabras clave: fenilcetonuria, inteligencia, función ejecutiva, fenilalanina.

a. Hospital

Universitario Río

Hortega, Servicio de Pediatría, Valladolid (España).

b. Universidad de León, Área de Personalidad, Evaluación y Tratamiento Psicológico.

Correspondencia: Dra. María B. González García, bgonzalezgarcia@ hotmail.com

Financiamiento: Ninguno.

Conflicto de intereses: Ninguno que declarar.

Recibido: 7-10-2016 Aceptado: 16-12-2016 mutaciones. ${ }^{1}$ Se produce por un déficit de la enzima hepática fenilalanina hidroxilasa, que convierte la fenilalanina en tirosina, con aumento de los niveles de fenilalanina y disminución de los de tirosina en la sangre y los tejidos. Esto provoca discapacidad intelectual si no se diagnostica y trata precozmente mediante dieta especial con bajo o nulo aporte de proteínas naturales y suplementos de aminoácidos libres de fenilalanina. ${ }^{2}$

Se diferencian dos grupos de pacientes: los que padecen fenilcetonuria y precisan tratamiento dietético para mantener niveles de fenilalanina dentro de los límites recomendables y los que padecen hiperfenilalaninemia benigna, que, por lo general, no precisan más que vigilancia periódica de las cifras de fenilalanina plasmática, pero sin tratamiento dietético.

El tratamiento se debe iniciar lo más precozmente posible y se recomienda mantenerlo de por vida debido a la relación existente entre el control dietético y el cociente intelectual (CI). ${ }^{3}$ Se recomienda iniciar tratamiento dietético en los pacientes que presentan valores de fenilalanina mayores de $6 \mathrm{mg} / \mathrm{dl}$ y vigilar con controles frecuentes a aquellos que presenten, en los primeros años de vida, valores de entre 2 y $6 \mathrm{mg} / \mathrm{dl}$ por si fuera necesario iniciar dieta por descompensaciones debidas a enfermedades propias de la edad. ${ }^{1}$ 
Las recomendaciones sobre los límites en los niveles de fenilalanina están siendo revisadas. Según la corriente americana, se deben mantener valores de entre 2 y $6 \mathrm{mg} / \mathrm{dl},{ }^{3}$ pero, según corrientes europeas, se deberían mantener valores menores de $4 \mathrm{mg} / \mathrm{dl} .{ }^{4}$ En lo que se está de acuerdo desde hace unos años es en la importancia de mantener estos niveles no solamente durante la infancia, sino durante toda la vida. ${ }^{5}$

También se observan déficits en las funciones ejecutivas, como planificación, organización y memoria operativa, a pesar de un buen control dietético, y se asocian, igualmente, los niveles de fenilalanina a los resultados en los tests que las evalúan. ${ }^{6}$ La velocidad de procesamiento se ve afectada en los pacientes con fenilcetonuria, lo cual parece relacionarse también con la afectación de las funciones ejecutivas. ${ }^{7}$

Por ello, es importante mantener los valores de fenilalanina en rangos de normalidad y controlar su variabilidad, ya que se ha observado que, a mayor variabilidad, peores resultados en el CI y las funciones ejecutivas. ${ }^{8,9}$

En este estudio, se analizó la relación entre los valores de fenilalanina plasmática y los resultados de diversos tests neuropsicológicos aplicados a pacientes con fenilcetonuria que seguían tratamiento dietético y pacientes con hiperfenilalaninemia benigna para evaluar su nivel de inteligencia y las funciones ejecutivas, y buscar una relación con los valores de fenilalanina plasmática.

\section{MATERIAL Y MÉTODOS}

Participantes: La muestra de pacientes del estudio fue extraída de la consulta de Metabolopatías del Hospital Universitario Río Hortega de Valladolid, hospital de tercer nivel y centro de referencia de enfermedades metabólicas para la comunidad de Castilla y León, que realiza el seguimiento y tratamiento a 20 pacientes con fenilcetonuria y a 26 con hiperfenilalaninemia benigna. De estos pacientes, se contactó con 26, que tenían edades entre 6 y 18 años (para que fuesen válidas las pruebas neuropsicológicas que se iban a realizar), de los cuales 16 padecían hiperfenilalaninemia benigna y 10 , fenilcetonuria. Todos accedieron a participar en el estudio. Todos los pacientes habían sido diagnosticados mediante pesquisa neonatal. La muestra de pacientes con fenilcetonuria consistía en 6 niñas y 4 niños de entre 10 y 18 años que habían sido tratados precozmente con dieta baja en proteínas y con la cual seguían en el momento del estudio. La muestra de pacientes con hiperfenilalaninemia benigna consistía en 6 niñas y 10 niños de entre 6 y 16 años que no precisaban dieta en el momento del estudio.

Intervenciones y medidas: Se realizó una revisión de las historias clínicas informatizadas para obtener los datos de niveles de fenilalanina a lo largo de la vida y se obtuvieron también los niveles de fenilalanina el día del estudio psicométrico (niveles de fenilalanina concurrente).

Los niveles de fenilalanina de los pacientes fueron determinados mediante cromatografía líquida de alta resolución (sistema cromatográfico UPLC Acquity acoplado a un detector de masas triple cuadrupolo Xevo TQ MS, fabricados por Waters Corporation, Milford, MA, USA). Se obtuvieron también los niveles de tirosina de los pacientes con fenilcetonuria y de 6 de los que presentaban hiperfenilalaninemia el día de los tests psicométricos mediante una muestra plasmática analizada por cromatografía líquida de alta resolución.

Para la estimación de los perfiles neuropsicológicos, se utilizaron la escala Wechsler de inteligencia para niños (Wechsler Intelligence Scale for Children; WISC-IV, por sus siglas en inglés), el test de copia de una figura compleja de A. Rey y el test de colores y palabras (Stroop) de Charles J. Golden. El instrumento de evaluación del estudio fue la prueba d2, test de atención.

La WISC-IV (2003, la adaptación a la población española 2005) está formada por 15 pruebas (10 principales y 5 opcionales) por las que se obtiene un perfil de puntuaciones escalares, un CI total y cuatro índices: comprensión verbal, razonamiento perceptivo, memoria de trabajo y velocidad de procesamiento. Es útil para evaluar dificultades de aprendizaje, funciones ejecutivas, lesiones cerebrales traumáticas, altas capacidades, discapacidad intelectual y otras alteraciones médicas y neurológicas.

Las escalas de Wechsler tienen una utilidad importante en el diagnóstico neuropsicológico. ${ }^{10,11}$ En niños con fenilcetonuria, han sido ampliamente utilizadas. ${ }^{2,8,12,13}$

El test de copia de una figura compleja de Rey es uno de los tests llamados "gestálticos", utilizados para el examen de las funciones cognitivas en pacientes con lesión cerebral o con trastorno por déficit de atención e hiperactividad. ${ }^{14}$ La tarea de esta prueba consiste en copiar primero y reproducir después, de memoria, una figura de estructura compleja. Muy utilizada con niños, aprecia el nivel del desarrollo perceptivo-motor, la atención y la memoria visual inmediata. 
El test de colores y palabras (Stroop) de Charles J. Golden (adaptación española de TEA ediciones) es un test atencional que se encarga de evaluar la habilidad para resistir la interferencia de tipo verbal, por lo que es una buena medida de la atención selectiva. ${ }^{15}$

El instrumento de evaluación del estudio fue la prueba d2, test de atención, elaborada por Rolf Brickenkamp. ${ }^{16}$ Este test supone una medida concisa de la atención selectiva y la concentración mental. Puede definirse la atención selectiva como "la capacidad para centrarse en uno o dos estímulos importantes, mientras se suprime deliberadamente la consciencia de otros estímulos distractores". ${ }^{17}$

Análisis estadístico: Los datos se analizaron mediante el paquete estadístico Stata ${ }^{\circledR} 12$ (Stata Corp., College Station, TX). Las variables continuas se expresaron como media y desviación estándar (DE) o como medianas y rango intercuartílico (interquartile range; IQR, por sus siglas en inglés), dependiendo de que presentasen distribución normal o no, respectivamente. Las variables categóricas se expresaron en forma de frecuencias absolutas y porcentajes. Se analizó, mediante correlación de Pearson, la relación entre los valores de fenilalanina en la sangre y los resultados de los tests psicométricos.

Normas éticas: El diseño del estudio fue aprobado por el Comité de Ética Asistencial y Ensayos Clínicos (CEIC) del Hospital Universitario Río Hortega, Área Oeste de Valladolid. Se obtuvo el consentimiento informado para la participación en el estudio y para la obtención de muestras biológicas destinadas a la investigación firmado por los propios pacientes o por sus tutores legales en caso de minoría de edad. En los menores de edad, asimismo, se obtuvo el asentimiento verbal por su parte para participar en el estudio.

\section{RESULTADOS}

Se analizaron datos de 26 pacientes, 10 con fenilcetonuria y 16 con hiperfenilalaninemia benigna. De aquellos diagnosticados con fenilcetonuria, 4 fueron varones, y la media de edad en años cumplidos fue de 12,5 (DE: 2,8). Entre los pacientes con hiperfenilalaninemia, 10 fueron varones, con una media de edad de 11,1 años cumplidos (DE: 2,8).

Para cada paciente, se calculó el $\mathrm{P}_{50}$ de sus valores de fenilalaninemia desde el diagnóstico. La media de estos valores entre los pacientes con fenilcetonuria fue de 5,72 mg/dl (DE: 1,13), mientras que, en los pacientes con diagnóstico de hiperfenilalaninemia, este valor fue de $3,57 \mathrm{mg} /$ dl (DE: 0,90$)(p<0,001)$. También se encontraron diferencias estadísticamente significativas entre ambos grupos en las medias de los valores de fenilalanina medida el día de la realización de los tests psicométricos; este valor fue de $9,70 \mathrm{mg} /$ dl (DE: 4,84) en los pacientes con fenilcetonuria, por $3,79 \mathrm{mg} / \mathrm{dl}(\mathrm{DE}: 1,20)$ en aquellos con hiperfenilalaninemia $(p<0,001)$. (Tabla 1)

Los valores de CI obtenidos mediante el test de Wechsler seguían una distribución normal con una media de 107 y una DE de 11,12.

En la Tabla 2, se observan los resultados de las pruebas de los tests neuropsicológicos en los pacientes con fenilcetonuria y en los pacientes con hiperfenilalaninemia, sin apreciarse diferencias estadísticamente significativas entre ambos, salvo en la puntuación del test d2, a favor del grupo de pacientes con hiperfenilalaninemia.

En la Tabla 3, figuran los coeficientes de correlación entre los valores sanguíneos de fenilalanina y el CI para cada grupo. Se observa una relación negativa entre los valores de fenilalanina el día de la realización de los tests y el CI. Esta relación es estadísticamente

TABLA 1. Características poblacionales y valores de fenilalanina en el grupo de pacientes con fenilcetonuria y en el grupo de pacientes con hiperfenilalaninemia benigna

\begin{tabular}{lcc}
\hline & Fenilcetonuria & $\begin{array}{c}\text { Hiperfenilalaninemia } \\
\text { benigna }\end{array}$ \\
\hline Total de pacientes & 10 & 16 \\
Edad media (DE) & $12,5(\mathrm{DE}: 2,8)$ & $11,1(\mathrm{DE}: 2,8)$ \\
Género (varones/mujeres) & $4 / 6$ & $10 / 6$ \\
Valores de fenilalanina desde el diagnóstico $(\mathrm{mg} / \mathrm{dl})$ & $5,72 \pm 1,13$ & $3,57 \pm 0,90$ \\
Valores de fenilalanina el día de los tests psicométricos $(\mathrm{mg} / \mathrm{dl})$ & $9,70 \pm 4,84$ & $3,79 \pm 1,20$ \\
\hline
\end{tabular}

DE: desviación estándar. 
significativa para el grupo de pacientes con hiperfenilalaninemia, no así para el grupo de pacientes con fenilcetonuria. No se han obtenido relaciones estadísticamente significativas entre el CI y la mediana de fenilalanina plasmática a lo largo de la vida de los pacientes o entre el CI $y$ el cociente entre fenilalanina y tirosina en el momento de la realización de los tests.

Se formaron grupos en nuestra muestra según los valores de fenilalanina concurrente el día de realización de los tests. En la Tabla 4, se ve que las medias de las distintas pruebas, si se comparan entre los que tienen $\geq 6 \mathrm{mg} / \mathrm{dl}$ y el resto, son claramente favorables al segundo grupo. Este hecho es explicable por el distinto grado de gravedad de la enfermedad de los 2 grupos de pacientes. El CI es menor en 8 puntos en el grupo de fenilalanina concurrente $\geq 6 \mathrm{mg} / \mathrm{dl}$ respecto al de $<6 \mathrm{mg} / \mathrm{dl}$.

En la Tabla 5, se ve la comparación de las medias de los distintos tests neuropsicológicos entre el grupo de pacientes con fenilalanina concurrente $<4 \mathrm{mg} / \mathrm{dl} \mathrm{y} \geq 4 \mathrm{mg} / \mathrm{dl}$. Se puede intuir una tendencia a tener mejores resultados en el grupo de menor fenilalanina el día de la prueba. Se observa que el CI también tiende a ser menor cuanto mayor sea el nivel de fenilalanina concurrente.

TABLA 2. Medias y desviaciones estándar de las puntuaciones percentiladas en los tests de evaluación neuropsicológica según el tipo de hiperfenilalaninemia

\begin{tabular}{llcc}
\hline Test & & Fenilcetonuria $\mathbf{( N = 1 0 )}$ & Hiperfenilalaninemia (N= 16) \\
\hline Escala de inteligencia de Wechsler & Cubos & $41,60 \pm 19,12$ & $45,13 \pm 29,80$ \\
& Semejanzas & $59,06 \pm 26,16$ & $74,56 \pm 19,33$ \\
& Dígitos & $45,00 \pm 27,20$ & $55,75 \pm 28,21$ \\
& Conceptos & $62,00 \pm 22,20$ & $61,88 \pm 25,59$ \\
& Claves & $69,90 \pm 23,00$ & $61,00 \pm 28,03$ \\
& Vocabulario & $73,30 \pm 26,90$ & $72,88 \pm 21,25$ \\
& Letras y números & $47,80 \pm 25,39$ & $53,31 \pm 25,09$ \\
& Matrices & $46,70 \pm 20,91$ & $53,19 \pm 29,06$ \\
& Comprensión & $47,86 \pm 29,10$ & $55,57 \pm 25,97$ \\
& Búsqueda de símbolos & $82,50 \pm 23,84$ & $64,91 \pm 27,11$ \\
& Figuras incompletas & $33,40 \pm 14,32$ & $28,44 \pm 17,30$ \\
& Animales & $75,40 \pm 22,33$ & $60,75 \pm 28,24$ \\
& Información & $55,59 \pm 24,94$ & $62,75 \pm 20,83$ \\
& Comprensión verbal & $62,77 \pm 28,02$ & $75,44 \pm 19,07$ \\
& Razonamiento perceptivo & $48,40 \pm 10,18$ & $53,13 \pm 29,53$ \\
\hline Test de atención d2 & Memoria de trabajo & $45,00 \pm 23,17$ & $53,85 \pm 28,15$ \\
Test de copia y reproducción de & Velocidad de procesamiento & $81,30 \pm 12,30$ & $67,75 \pm 27,28$ \\
\hline una figura compleja de Rey & Comisiones & $15,10 \pm 8,63$ & $33,31 \pm 27,84$ \\
\hline Test de colores y palabras de Stroop & Resistencia interferencia & $4,19 \pm 5,44$ & $88,63 \pm 15,13$ \\
\hline & & & $58,94 \pm 29,33$ \\
\hline & Tiempo & $80,60 \pm 29,89$ & $51,19 \pm 29,70$ \\
\hline
\end{tabular}

TABla 3. Valores del coeficiente de correlación de Pearson entre los valores sanguíneos de fenilalanina y tirosina y el cociente intelectual

\begin{tabular}{lcc}
\hline & HPB (N= 16) & PKU (N = 10) \\
\hline Fenilalanina concurrente $^{1}$ & $\mathbf{- 0 , 6 0 8}$ & $-0,060$ \\
Mediana de fenilalanina $^{2}$ & $-0,232$ & 0,131 \\
Fenilalanina/tirosina $^{3}$ & $-0,273$ & 0,329 \\
\hline
\end{tabular}

* En negrita, están señalados los valores que presentaron un valor de $\mathrm{p}<0,05$.

HPB: pacientes diagnosticados de fenilalaninemia benigna; PKU: pacientes con fenilcetonuria.

NOTA: ${ }^{1}$ Fenilalaninemia en el momento de realización del test; ${ }^{2}$ Mediana de los valores históricos de fenilalanina de cada paciente; ${ }^{3}$ Cociente entre los valores sanguíneos de fenilalanina y tirosina en el momento de realización del test (el cociente fenilalanina/tirosina solo se determinó en 6 pacientes del grupo HPB). 


\section{DISCUSIÓN}

En nuestra muestra, hay un predominio de pacientes con hiperfenilalaninemia, en una proporción similar a la que ocurre en la población española, que contrasta con lo que sucede en los países norteuropeos, donde predominan las

Tabla 4. Medias y desviaciones estándar de las puntuaciones en los tests de evaluación neuropsicológica en el grupo de pacientes con fenilalanina concurrente menor de $6 \mathrm{mg} / \mathrm{dl}$ o mayor de $6 \mathrm{mg} / \mathrm{dl}$ o igual el día de realización de los tests

\begin{tabular}{|c|c|c|c|}
\hline Test & Fenil & Inina $<6 \mathrm{mg} / \mathrm{dl}(\mathrm{N}=17)$ & Fenilalanina $\geq 6 \mathrm{mg} / \mathrm{dl}(\mathrm{N}=9)$ \\
\hline Escala de inteligencia de Wechsler & $\begin{array}{l}\text { Cubos } \\
\text { Semejanzas } \\
\text { Dígitos } \\
\text { Conceptos } \\
\text { Claves } \\
\text { Vocabulario } \\
\text { Letras y números } \\
\text { Matrices } \\
\text { Comprensión } \\
\text { Búsqueda de símbolos } \\
\text { Figuras incompletas } \\
\text { Animales } \\
\text { Información } \\
\text { Comprensión verbal } \\
\text { Razonamiento perceptivo } \\
\text { Memoria de trabajo } \\
\text { Velocidad de procesamiento } \\
\text { CI }\end{array}$ & $\begin{array}{r}47,53 \pm 27,49 \\
72,15 \pm 22,80 \\
56,71 \pm 26,62 \\
61,12 \pm 26,61 \\
67,65 \pm 24,47 \\
72,35 \pm 22,08 \\
54,59 \pm 24,86 \\
53,77 \pm 28,24 \\
57,45 \pm 27,01 \\
66,15 \pm 28,17 \\
29,71 \pm 17,55 \\
63,47 \pm 28,73 \\
61,76 \pm 21,99 \\
74,52 \pm 20,35 \\
53,82 \pm 27,42 \\
55,09 \pm 27,16 \\
71,59 \pm 23,00 \\
109,29 \pm 12,17 \\
\end{array}$ & $\begin{array}{l}36,67 \pm 22,09 \\
61,89 \pm 21,19 \\
42,00 \pm 28,92 \\
63,44 \pm 23,81 \\
58,33 \pm 29,45 \\
74,33 \pm 26,13 \\
44,78 \pm 24,95 \\
44,89 \pm 21,33 \\
43,44 \pm 25,67 \\
82,11 \pm 21,91 \\
31,56 \pm 13,87 \\
71,89 \pm 22,76 \\
56,67 \pm 23,80 \\
63,11 \pm 27,66 \\
46,56 \pm 15,06 \\
41,67 \pm 23,28 \\
75,56 \pm 25,25 \\
102,67 \pm 7,63 \\
\end{array}$ \\
\hline Test de atención d2 & Comisiones & $14,56 \pm 7,45$ & $35,53 \pm 27,38$ \\
\hline $\begin{array}{l}\text { Test de copia y reproducción de } \\
\text { una figura compleja de Rey }\end{array}$ & $\begin{array}{l}\text { Copia } \\
\text { Tiempo } \\
\text { Memoria }\end{array}$ & $\begin{array}{c}91,59 \pm 8,30 \\
58,41 \pm 28,49 \\
50,24 \pm 29,76\end{array}$ & $\begin{array}{l}74,11 \pm 33,49 \\
60,11 \pm 36,25 \\
58,56 \pm 29,15\end{array}$ \\
\hline
\end{tabular}

CI: cociente intelectual.

TABla 5. Medias y desviaciones estándar de las puntuaciones en los tests de evaluación neuropsicológica en el grupo de pacientes con fenilalanina concurrente menor de $4 \mathrm{mg} / \mathrm{dl}$ o mayor de $4 \mathrm{mg} / \mathrm{dl}$ o igual el día de realización de los tests

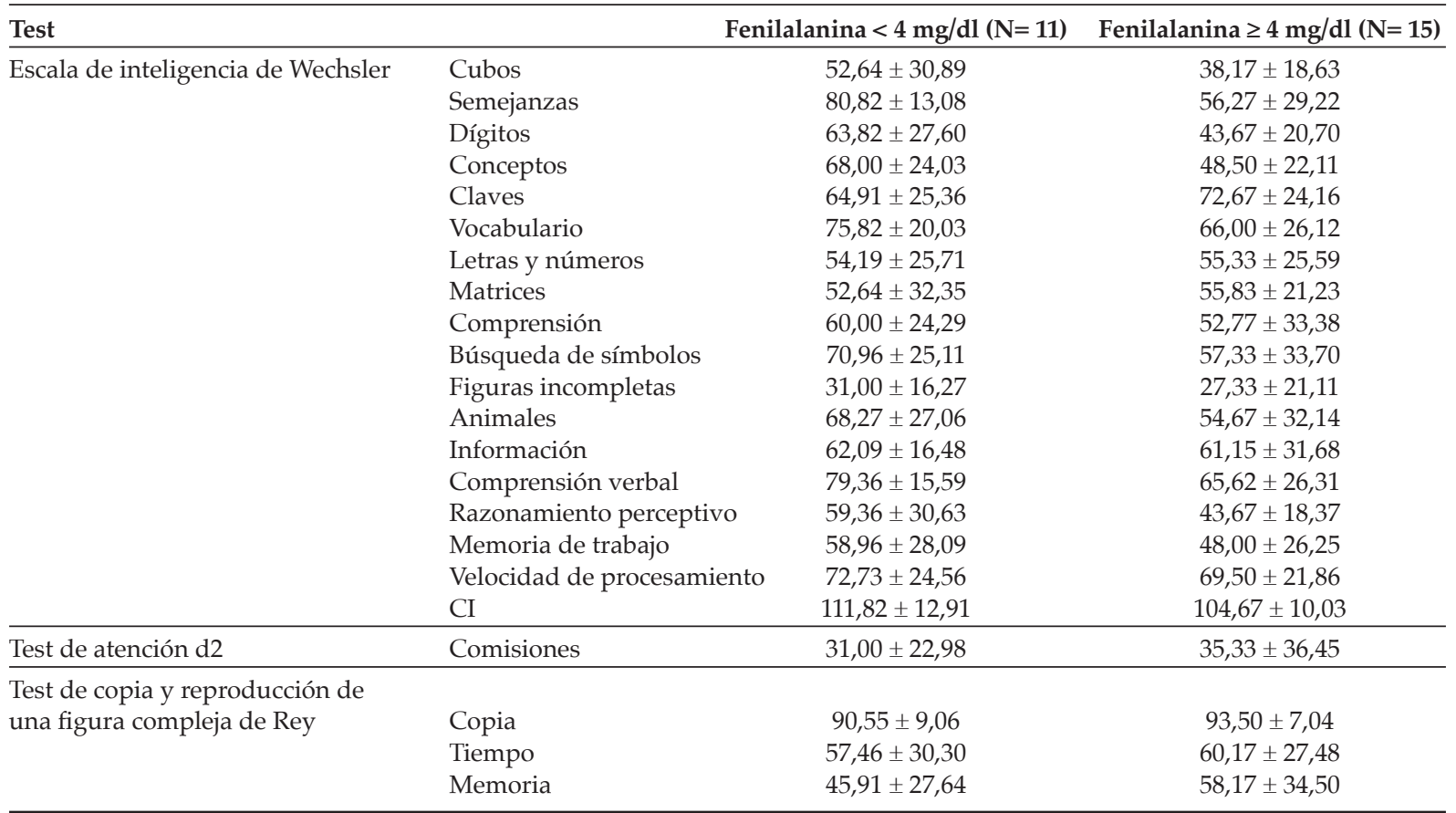

CI: cociente intelectual. 
formas más graves. ${ }^{18}$

En varios estudios, se ha observado que, con el diagnóstico precoz de la fenilcetonuria y su inicio precoz de tratamiento dietético, los pacientes pueden presentar una función intelectual dentro de valores normales, aunque en límites levemente más bajos que controles sanos. ${ }^{4,19} \mathrm{~A}$ diferencia de estos estudios, en nuestro grupo de pacientes, se observa un $\mathrm{CI}$ en valores ligeramente superiores a la normalidad.

También se ha demostrado que los pacientes con hiperfenilalaninemia tienen una incidencia de problemas neuropsicológicos mayores que en la población sana. ${ }^{20,21}$

Existe una relación entre la función intelectual y los valores de fenilalanina plasmática a lo largo de la vida, sobre todo, en períodos críticos de desarrollo neurológico, pero también se ha observado relación con los valores concurrentes de fenilalanina en el momento de la realización de los tests. ${ }^{12,22}$ En nuestro grupo de pacientes, hay una tendencia a la relación inversa entre el CI y la fenilalanina concurrente, la mediana de fenilalanina y el cociente fenilalanina/tirosina.

Además de evaluar la función intelectual mediante el CI, cada vez, se da más importancia a los déficits en las funciones ejecutivas, ya que, aunque tengan CI normales, pueden presentar dificultades en la vida diaria por estas limitaciones en ciertas funciones ejecutivas. ${ }^{13.23}$

Las puntuaciones en los tests que evalúan funciones ejecutivas se relacionan negativamente con los valores de fenilalanina plasmática. ${ }^{24,25}$ En nuestros pacientes, se detecta una tendencia a la relación negativa entre la mayoría de las funciones ejecutivas evaluadas y los valores de fenilalanina concurrentes y a lo largo de la vida.

Como ya se ha descrito en otros estudios, hay diferencias en cuanto a los resultados en las funciones ejecutivas, que son peores en pacientes con hiperfenilalaninemia sin dieta que en pacientes con fenilcetonuria en tratamiento dietético precoz. ${ }^{20}$ Esto lleva a pensar que no es suficiente con mantener los valores de fenilalanina por debajo de ciertos límites, sino que habría que procurar que fueran lo más bajos posible.

Los estudios más recientes sobre el umbral de fenilalaninemia para iniciar tratamiento se basan en los diferentes resultados que se obtienen en los pacientes con niveles de fenilalanina por debajo de $4 \mathrm{mg} / \mathrm{dl}$ y los pacientes entre 4 y $6 \mathrm{mg} /$ dl. ${ }^{4}$ En nuestro grupo de pacientes, se muestra una tendencia de mejorar los resultados en las pruebas de la función ejecutiva de forma lineal al disminuir los niveles de fenilalanina; este hecho es más marcado en los pacientes sin tratamiento, formas benignas. A la luz de estos resultados, nuestros datos apoyan la idea ya propuesta por otros autores de disminuir los límites aceptables de fenilalanina plasmática.

El concepto clásico del objetivo de tratamiento en fenilcetonuria ha ido cambiando con los diversos estudios realizados a lo largo del tiempo: desde el año 2000, cuando se introduce la idea de la "diet for life", ${ }^{26,27}$ hasta los trabajos actuales, que indican que los objetivos del tratamiento deben ser intentar conseguir el máximo potencial de la capacidad intelectual y, como se ve en nuestros resultados, que las elevaciones de fenilalanina puntuales también se correlacionan con un menor rendimiento en los tests de la función ejecutiva. ${ }^{9}$ Esto nos introduce la idea de que una dieta más estricta de forma puntual podría mejorar los resultados en la vida diaria de nuestros pacientes. En momentos de necesidad de un mayor rendimiento intelectual, un control con menores niveles de fenilalaninemia debería ir asociado a mejores resultados en la vida diaria, como el rendimiento laboral o académico. Sería necesario realizar más estudios que evaluaran la influencia de los valores de fenilalanina plasmática en la vida diaria de los pacientes, así como en cuanto a la salud mental y las funciones sociales, ya que se han visto diferencias respecto a sujetos sanos..$^{28,29}$ Sería interesante estudiar los beneficios de este control más estricto para mantener los valores de fenilalanina lo más bajos posible en pacientes con hiperfenilalaninemias en épocas que requieran alto rendimiento intelectual, como exámenes.

Se puede afirmar que no solo es importante evitar aumentos mantenidos de fenilalanina plasmática, sino que los aumentos puntuales pueden también provocar alteraciones neuropsicológicas, y que hay un nuevo horizonte abierto respecto a la disminución de los valores aceptables de fenilalanina plasmática para alcanzar el máximo potencial intelectual de estos pacientes.

No es sencillo poder realizar baterias de tests psicológicos tan exhaustivas como las de este estudio, que, además, han sido todas aplicadas por el mismo equipo neuropsicológico, lo que aumenta su validez interna.

\section{CONCLUSIONES}

Nuestros pacientes presentan un CI en rangos de normalidad con tendencia a la relación con los niveles de fenilalanina plasmática. 
Se aprecia una relación negativa entre la mayoría de las funciones ejecutivas evaluadas y los valores de fenilalanina concurrentes y a lo largo de la vida.

\section{REFERENCIAS}

1. Vockley J, Andersson HC, Antshel KM, Braverman NE, et al. Phenylalanine hydroxylase deficiency: diagnosis and management guideline. Genet Med 2014;16(2):188-200.

2. Vilaseca MA, Lambruschini N, Gómez-López L, Gutiérrez A, et al. Quality of dietary control in phenylketonuric patients and its relationship with general intelligence. Nutr Hosp 2010;25(1):60-6.

3. Singh RH, Rohr F, Frazier D, Cunningham A, et al. Recommendations for the nutrition management of phenylalanine hydroxylase deficiency. Genet Med 2014;16(2):121-31.

4. Jahja R, Huijbregts SC, de Sonneville LM, van der Meere $\mathrm{JJ}$, et al. Neurocognitive evidence for revision of treatment targets and guidelines for phenylketonuria. J Pediatr 2014;164(4):895-9.e2.

5. Demirkol M, Giżewska M, Giovannini M, Walter J. Follow up of phenylketonuria patients. Mol Genet Metab 2011;104(Suppl):S31-9.

6. Crossley LH, Anderson PJ. Función neuropsicológica en la fenilcetonuria tratada precozmente: una revisión. Ann Nestlé (Esp) 2010;68(2):79-89.

7. Janos AL, Grange DK, Steiner RD, White DA. Processing speed and executive abilities in children with phenylketonuria. Neuropsychology 2012;26(6):735-43.

8. Hood A, Grange DK, Christ SE, Steiner R, et al. Variability in phenylalanine control predicts IQ and executive abilities in children with phenylketonuria. Mol Genet Metab 2014;111(4):445-51.

9. Cleary M, Trefz F, Muntau AC, Feillet F, et al. Fluctuations in phenylalanine concentrations in phenylketonuria: a review of possible relationships with outcomes. Mol Genet Metab 2013;110(4):418-23.

10. Manga D, Fournier C. Neuropsicología clínica infantil: Estudio de casos en edad escolar. Madrid: Universitas; 1997.

11. Conde-Guzon PA, Cancho-Candela R. Epilepsia generalizada idiopática con ausencias típicas tratadas con ácido valproico: alteraciones neuropsicológicas. Rev Neurol 2012;55(2):65-73.

12. Cappelletti S, Cotugno G, Goffredo BM, Nicolò R, et al. Cognitive findings and behavior in children and adolescents with phenylketonuria. J Dev Behav Pediatr 2013;34(6):392-8.

13. Viau KS, Wengreen HJ, Ernst SL, Cantor NL, et al. Correlation of age-specific phenylalanine levels with intellectual outcome in patients with phenylketonuria. $J$
Inherit Metab Dis 2011;34(4):963-71.

14. Rey A. REY, Test de copia y de reproducción de memoria de figuras geométricas complejas. $9^{\mathrm{a}}$ ed.Madrid:TEA; 2009.

15. Golden CJ.Stroop: test de colores y palabras. $5^{\mathrm{a}}$ ed. Madrid: TEA; 2010.

16. Brickenkamp R. Test de atención: d2. Madrid: TEA; 2002.

17. Jiménez JE, Hernández S, García E, Díaz A, et al. Test de atención D2: Datos normativos y desarrollo evolutivo de la atención en educación primaria. Eur J Educ Psychol 2012;5(1):93-106.

18. Arena J, Cortés E, Dulín E, Eguileor I, et al. Actividad de los centros de detección precoz neonatal de errores congénitos del metabolismo en España en el período 2000-2008. Boletín del Real Patronato de Discapacidad 2010;69:14-30.

19. Weglage J, Fromm J, Van Teeffelen-Heithoff A, Möller $\mathrm{HE}$, et al. Neurocognitive functioning in adults with phenylketonuria: results of a long term study. Mol Genet Metab 2013;110(Suppl):S44-8.

20. Campistol J, Gassió R, Artuch R, Vilaseca MA. Neurocognitive function in mild hyperphenylalaninemia. Dev Med Child Neurol 2011;53(5):405-8.

21. Gassió R, Artuch R, Vilaseca MA, Fusté E, et al. Cognitive functions in classic phenylketonuria and mild hyperphenylalaninaemia: experience in a paediatric population. Dev Med Child Neurol 2005;47(7):443-8.

22. Castro IP, Borges JM, Chagas HA, Tibúrcio J, et al. Relationships between phenylalanine levels, intelligence and socioeconomic status of patients with phenylketonuria. J Pediatr (Rio J) 2012;88(4):353-6.

23. Christ SE, Huijbregts SC, de Sonneville LM, White DA. Executive function in early-treated phenylketonuria: profile and underlying mechanisms. Mol Genet Metab 2010;99(Suppl 1):S22-32.

24. Antenor-Dorsey JA, Hershey T, Rutlin J, Shimony JS, et al. White matter integrity and executive abilities in individuals with phenylketonuria. Mol Genet Metab 2013;109(2):125-31.

25. Huijbregts SC, Gassió R, CampistolJ. Executive functioning in context: Relevance for treatment and monitoring of phenylketonuria. Mol Genet Metab 2013;110(Suppl):S25-30.

26. Brenton DP, Pietz J. Adult care in phenylketonuria and hyperphenylalaninaemia: the relevance of neurological abnormalities. Eur J Pediatr 2000;159(Suppl 2):S114-20.

27. Phenylketonuria (PKU): screening and management. NIH Consens Statement 2000;17(3):1-33.

28. Jahja R, Huijbregts SC, de Sonneville LM, van der Meere JJ, et al. Mental health and social functioning in early treated Phenylketonuria: thePKU-COBESO study.Mol Genet Metab 2013;110(Suppl):S57-61.

29. Banerjee P, Grange DK, Steiner RD, White DA. Executive strategic processing during verbal fluency performance in children with phenylketonuria. Child Neuropsychol 2011;17(2):105-17. 